\title{
Plenary Lectures
}

Huge progresses have been made those last years concerning different aspects of pancreatic development. First, a large number of transcription factors have been shown to be crucial for pancreatic development both in rodent and human. Next, different mechanisms have been shown to be implicated in the control of pancreatic development. It has been shown that at early stages, signals derived from the notochord do control the development of the dorsal pancreas. Later on, signals derived from the pancreatic mesenchyme that surrounds the epithelium do control the proliferation of immature epithelial cells and their differentiation into endocrine or acinar cells. To progress in the characterization of those specific factors, we are designing simple experimental systems in rodent and human, which allow studying the implication of specific factors in the proliferation, differentiation and morphogenesis of the pancreas. Results obtained with those experimental systems will be presented.

\section{PL1-1 \\ ER- $\alpha$ and ER- $\beta$ Knock Out Mouse Models: Relevance to Human Physiology and Pathology \\ J.F. Couse ${ }^{1}$, K.S. Korach ${ }^{1}$ \\ 1 Laboratory of Reproductive and Developmental Toxicoloy, National Institute of Environmental Health Sciences, NIH, Research Triangle Park, NC, USA}

The majority of documented estrogen actions require the presence of functional estrogen receptor (ER) within the nuclei of target cells. The ER is known to exist in two forms, ER $\alpha$ and ER $\beta$, and fulfill critical roles as ligand-inducible transcription factors in the physiology and function of multiple systems. Although in vitro studies suggest the two ERs may play redundant roles, a dissimilar tissue distribution indicates otherwise. To study the role of each ER, individual lines of mice that lack each individual ER as well as both were generated. The $\alpha$ ERKO and $\beta E R K O$ mice survive to adulthood, exhibit normal development of the reproductive tract and maintain expression of the opposite ER. The $\alpha$ ERKO female is infertile, exhibiting a hypoplastic uterus, anovulatory, polycystic ovaries and elevated serum levels of estradiol, testosterone and $\mathrm{LH}$. The $\alpha \mathrm{ERKO}$ ovarian phenotype and hormonal milieu is comparable to polycystic ovarian syndrome (PCOS) in humans. Treatment of $\alpha E R K O$ females with a GnRH antagonist is able to prevent the onset of polycystic ovaries, indicating that chronic LH stimulation due to the loss of ER $\alpha$ in the hypothalamic-pituitary axis is the primary cause of this phenotype, rather than the disruption of intraovarian ER $\alpha$ actions. In contrast, the $\beta E R K O$ female exhibits a sexually mature uterus, relatively normal ovaries and serum hormone levels; however is sub-fertile, characterized by reductions in the frequency of pregnancy and litter size. In males, the importance of estrogen action is clearly indicated by complete infertility in the $\alpha E R K O$ due to abnormalities in spermatogenesis and behavior; whereas, $\beta E R K O$ males show no deficits in fertility. Mice possessing a disruption of both ER genes $(\alpha \beta E R K O)$ show no marked external abnormalities and exhibit the expected reproductive tract structures, yet are infertile. Remarkably, the ovaries of $\alpha \beta E R K O$ females are characterized by a dramatic postnatal loss of oocytes and the apparent redifferentiation of the remaining somatic cells to Sertoli-like cells, including ectopic expression of the Sox 9 gene. Several phenotypes observed in the ERKO mice are comparable to those that are described in the few reported cases of estrogen insufficiency and the single known case of estrogen resistance among humans. Therefore, the continued characterization and use of the ERKO mice should provide great insight toward the verification of the separate as well as the likely cooperative roles the two ERs play in normal physiology as well as infertility, carcinogenesis and toxicology.

\section{PL3-3 \\ Assisted Reproductive Technologies (ART) and Pre-Implantation Genetics \\ André Van Steirteghem ${ }^{1}$ \\ ${ }^{1}$ Centre for Reproductive Medicine, Vrije Universiteit Brussels, Belgium}

In the last two decades two ART procedures have revolutionised the treatment of longstanding infertility. Since the birth of Louise Brown (July 1978) in-vitro fertilization can alleviate the majority of cases with female-factor infertility (e.g. tubal disease and severe endometriosis). In 1992 our group reported the first births to result from intracytoplasmic (single) sperm injection (ICSI) in couples with severe male-factor infertility. By now ICSI can be used successfully in couples with severe male-factor infertility including a number of patients with azoospermia. In order to evaluate the safety of IVF as well as ICSI a prospective follow-up study of children born after IVF and ICSI was carried out and is still ongoing. At birth the majority $(\sim 97 \%)$ of children are healthy, the incidence of major congenital malformations in ICSI children is similar to the incidence in IVF children. The progress of micro-manipulation techniques within the field of assisted reproduction and the development of molecular genetic knowledge and technology resulted in the development of preimplantation genetic diagnosis (PGD), changing the options for couples with a genetic risk. PGD can be considered an extremely early form of prenatal diagnosis (PND), in which the disease is diagnosed at the embryonic level in vitro before transfer and before pregnancy. Hence the physical and psychological trauma associated with possible termination of pregnancy is avoided. Standard PGD is carried out in four phases, including ART phases and genetic phases. Five years' experience of preimplantation genetic diagnosis (PGD) at the Brussels Free University will be reviewed. Patients were treated for autosomal recessive, autosomal dominant and X-linked recessive monogenic disorders as well as for autosomal structural aberrations, sex chromosome numerical and structural aberrations and a combination of the two latter. Specific diagnosis was carried out by polymerase chain reaction (PGD) or fluorescence in situ hybridization (FISH) was used for sexing. Our series demonstrate that PGD is a feasible technique by which to avoid the birth of genetically affected children to couples at risk.

\section{KARGER \\ Fax + 41613061234 \\ E-Mail karger@karger.ch}

www. karger.com

\section{(c) 2000 S. Karger AG, Basel}

Accessible online at:

www. karger.com/journals/hre 


\section{PL4-4}

\section{The Impact of Molecular Biology on Clinical \\ Endocrinology: Past, Present and Future}

G.P. Chrousos ${ }^{1}$

${ }^{1}$ Pediatric and Reproductive Endocrinology Branch, NICHD, NIH,

Bethesda, MD, USA

Immense, parallel progress has taken place in Biology, Electronic Computation and Nanomechanics/Robotics in the last 20 years. Molecular, Cell, and Developmental Biology are adding daily to human knowledge at exponential speeds. The human body consists of about 10 trillion cells of over 200 distinct types, while the human genome expresses over 80,000 genes, of which at least 30,000 are expressed in the brain. Not too long from today the entire sequence of the human genome of the 3 billion bases will be available on line. This overwhelming knowledge has resulted in the development of new concepts, with interlinked genes or cells forming functional modules, the latter inter-linked into networks. Genomics, transcriptomics, proteomics, physiomics, and biomics are new terms that represent new fields. Major biomedical research centers are gearing up for characterizing individual genes as to their distribution of expression in the body, homologies with other species, chromosomal mapping, normal and abnormal functions, polymorphisms, and clinical relevance. At this time, the genes responsible for many monogenic disorders, including several types of cancer, have been identified, as well as the pathogenic molecular lesions; this will be expanded within the next few years to include the majority of disease-causing defects. Major concepts on the pathogenesis of frequent, heritable polygenic disorders that plague humanity, such as obesity and hypertension, have been developed. These disorders reflect selective pressures on the genome of Homo sapiens sapiens and his/her ancestors whose purpose was to defend the organism against threats such as starvation and dehydration. The diversity of the multiple genes that are involved in the heritability of these disorders is physiologic and frequently due to single nucleotide polymorphisms within the regulatory or structural areas of genes. These quantitative traits define different amounts of expression and/or activity of these genes, and their presence is neither necessary nor sufficient for the expression of the polygenic disorder. Rather, their effects are epistatic and their presence defines a probable risk for development of a disorder rather than the disorder itself. The great majority of cancers are somatic polygenic disorders involving multiple defective genes. In the next few years the transcriptomics and proteomics of most polygenic disorders will be defined and we will be able to identify risks for conditions such as obesity, hypertension, autoimmune disorders, anxiety, and depression. We will also define the exact genetic defects of cancers and devise ways to intervene. Stem cell therapy and gene therapy or a combination of the two will hopefully be a reality and many monogenic disorders will become curable. Preimplantation diagnosis and gene therapy will allow prevention of such disorders. Cancer therapy will be individualized to the patient's cancer, which will also hopefully be curable. The cloning of receptors, enzymes and other classes of molecules has already allowed the development of new plarmacologic agents for many pathologic conditions. This will continue, with the discovery of an ever expanding array of medicines whose effect will be as specific and as innocuous as possible. 